\title{
Authors' reply to Grant and Garland and to Bolland and colleagues
}

\author{
Rajiv Chowdhury cardiovascular epidemiologist ${ }^{1}$, Oscar H Franco professor ${ }^{2}$, On behalf of Setor \\ Kunutsor, Anna Vitezova, Clare Oliver-Williams, Susmita Chowdhury, Jessica C Kiefte-de-Jong, \\ Hassan Khan, Cristina P Baena, Dorairaj Prabhakaran, Moshe B Hoshen, Becca S Feldman, An \\ Pan, Laura Johnson, Francesca Crowe, and Frank B Hu
}

${ }^{1}$ Department of Public Health and Primary Care, University of Cambridge, Strangeways Research Laboratory, Cambridge CB1 8RN, UK; ${ }^{2}$ Department of Epidemiology, Erasmus MC, Rotterdam, Netherlands

We agree with Grant and Garland that, although existing ecological studies support the findings of our meta-analysis of observational studies, further work-especially that involving well powered randomised intervention studies-is needed. ${ }^{12}$ However, the respective pooled risk ratios that we reported by combining the primary and secondary prevention cohorts are based on indirect comparisons (only a subset of studies provided mortality risk data on people with pre-existing disease).

As Bolland and colleagues note, the overall estimates from the vitamin $\mathrm{D}_{3}$ randomised controlled trials were indeed presented as a combination of both active and inactive vitamin $D_{3}$ supplements, given a lack of power in each component in isolation. ${ }^{3}$ We also included a study that evaluated the effects of vitamin $\mathrm{D}_{3}$ alone without concurrent administration of other pharmacological interventions (which was similarly kept as a vitamin D alone study in the earlier Cochrane report). ${ }^{14}$ Nonetheless, when this study and the other three calcitriol trials were removed from the analyses, ${ }^{5-7}$ there was no significant effect of "any vitamin D supplementation" on mortality (which remains consistent with our original results). The pooled effect estimate for the 10 vitamin $\mathrm{D}_{3}$ trials became slightly attenuated $(0.91,95 \%$ CI 0.82 to 1.00$)$ in our calculation; however, this apparent inverse effect differed significantly from the corresponding pooled estimate of vitamin $\mathrm{D}_{2}(\mathrm{P}$ from meta-regression analysis $=0.03$, for a comparison between vitamin $\mathrm{D}_{3}$ and vitamin $\mathrm{D}_{2}$ trials). That said, we agree with Bolland and colleagues that the selection criteria (such as randomised $v$ non-randomised, with calcium supplementation $v$ without) and decisions on subgroup analyses vary across reviews on this topic, and this may explain the different findings across these reports. However, as was discussed in our paper (and the accompanying editorial), all these reviews (including ours) are based on largely overlapping trials of mostly high risk, elderly populations (with an average age $>75$ years in all trials combined). Therefore, before any policy formulation, further large scale and sufficiently prolonged trials with large samples derived from the general population will be required.

\section{Competing interests: None declared.}

Chowdhury R, Kunutsor S, Vitezova A, Oliver-Williams C, Chowdhury S, Kiefte-de-Jong $\mathrm{JC}$, et al. Vitamin $\mathrm{D}$ and risk of cause specific death: systematic review and meta-analysis of observational cohort and randomised intervention studies. BMJ 2014;348:g1903. (1 April.)

2 Grant WB, Garland CF. Vitamin D has a greater impact on cancer mortality rates than on cancer incidence rates. BMJ 2014;348:g2862.

3 Bolland MJ, Grey A, Reid IR. Vitamin D supplements do not reduce mortality risk. BMJ 2014;348:g2860.

4 Campbell AJ, Robertson MC, La Grow SJ, Kerse NM, Sanderson GF, Jacobs RJ, et al. Randomised controlled trial of prevention of falls in people aged $\geq 75$ with severe visual impairment: the VIP trial. BMJ 2005;331:817.

5 Grady D, Halloran B, Cummings S, Leveille S, Wells L, Black D, et al. 1,25-dihydroxyvitamin $D_{3}$ and muscle strength in the elderly: a randomized controlled trial. J Clin Endocrinol Metab 1991;73:1111-7.

6 Gallagher JC. The effects of calcitriol on falls and fractures and physical performance tests. J Steroid Biochem Mol Biol 2004;89-90:497-501.

7 Beer TM, Ryan CW, Venner PM, Petrylak DP, Chatta GS, Ruether JD, et al. Double-blinded randomized study of high-dose calcitriol plus docetaxel compared with placebo plus docetaxel in androgen-independent prostate cancer: a report from the ASCENT Investigators. J Clin Oncol 2007;25:669-74. 